\title{
STRING GONIOMETRY BY NEUTRAL CURRENTS 하
}

\author{
F. DEL ÁGUILA ${ }^{\text {a.b }}$, J.A. GONZÁLEZ ${ }^{\mathrm{b}}$ and M. QUIRÓS ${ }^{\mathrm{a}, \mathrm{c}}$ \\ a University of Michigan, Ann Arbor, MI 48109, USA \\ b Department de Fisica Teòrica, UAB, Bellaterra, E-08193 Barcelona, Spain \\ c CSIC, Serrano 119, E-28006 Madrid, Spain
}

Received 8 July 1987

\begin{abstract}
If a new neutral gauge boson exists at accelerator energies, the measurement of the mixing angle(s) fixing its current could to a large extent determine the (heterotic) string model at the Planck scale. In this paper we present the renormalization group predictions for this angle.
\end{abstract}

String theories seem to be a consistent framework to unify gravity and chiral gauge theories [1]. In this respect, the $E_{8} \times E_{8}^{\prime}$ heterotic string [2] appears to be rather unique. Since it is formulated in ten dimensions, the six extra dimensions must compactify and the gauge group reduce in order to make contact with the observed world. Four-dimensional string theories may be seen as special subcases [3]. In any case, a crucial point is what are the gauge interactions at low energies. Calabi-Yau manifold or orbifold compactifications allow extended electroweak models, as does experiment. Here we concentrate on the possibility of one extra gauge boson at accelerator energies.

It must be emphasized that independently of the details of the compactification, there are only two possible choices for the corresponding extra $U_{1}$. In any case, one of them must always accompany the last step of gauge symmetry breaking down to the standard model (SM). This follows from the gauge content of the heterotic string, from which all quantum numbers derive. The argument goes as follows. If the SM must contain the standard chiral fermions, it must be possible to embed it in the standard $\mathrm{SU}_{5}$, which can be identified with one of the $\mathrm{SU}_{5}$ of the maximal $\mathrm{E}_{8}$ subgroup $\mathrm{SU}_{5} \times \mathrm{SU}_{5}^{\prime}$. Under this, the gauge representation decomposes as

tr Work partially supported by the US-Spain Joint Committee for Scientific and Technological Cooperation and the CAICYT.

$$
\begin{aligned}
248 & =(24,1)+(1,24)+(10,5) \\
& +(\overline{10}, \overline{5})+(5, \overline{10})+(\overline{5}, 10) .
\end{aligned}
$$

Independently of the mechanism of gauge symmetry breaking, the only directions along which the vacuum can align without breaking $\mathrm{SU}_{3}^{\mathrm{C}} \times \mathrm{SU}_{2}^{\mathrm{L}} \times \mathrm{U}_{1}^{Y}$, are the standard $\mathrm{SU}_{5}$ direction along the 24 and all the other directions in the 24 of the orthogonal $\mathrm{SU}_{5}^{\prime}$. Proton decay requires the standard $\mathrm{SU}_{5}$ to be broken at the compactification scale. Then, we must only deal with the 24 of the extra $\mathrm{SU}_{5}^{\prime}$. The directions along which the vacuum aligns define the unbroken $\mathrm{SU}_{5}^{\prime}$ subgroup $\mathrm{H}$, which in turn defines the $\mathrm{SU}_{5}^{\prime}$ subgroup associated to the vacuum directions, $\mathbf{H}^{\prime}$,

$\left[h, h^{\prime}\right]=0, \quad \forall h^{(\prime)} \in \mathrm{H}^{(\prime)}$.

For vacuum alignment along the adjoint representation, $\mathrm{H}^{\prime}$ must be some piece(s) of a maximal subgroup of the extra $\mathrm{SU}_{5}^{\prime}, \mathrm{SU}_{3} \times \mathrm{SU}_{2} \times \mathrm{U}_{1}$ or $\mathrm{SU}_{4} \times \mathrm{U}_{1}$. Then $\mathrm{H}$ must necessarily contain one of those $U_{1}$. Note that this is independent of the explicit realization of the $\mathrm{E}_{8}$ or $\mathrm{SU}_{5}$ gauge symmetry, and of the scales of symmetry breaking. The last step of symmetry breaking down to the SM must contain one of these two $U_{1}$ 's. Then if only one extra $U_{1}$ exists at accelerator energies, it must be one of these. Needless to say, that since the $U_{1}$ 's of the extra $E_{8}^{\prime}$ do not couple to the standard fermions, they are not relevant for our discussion. Besides, gauge invariance is 
defined by zero modes and its breaking is associated only to the vacuum alignment along the adjoint representation in (1).

The first $U_{1}$ is that of the minimal model, $Y^{\prime}$, and the other is $B-L$ (see below). Interestingly enough, both $U_{1}$ are included in $E_{6}[4]^{\# 1}$ :

$$
\begin{aligned}
\mathrm{E}_{8} & \supset\left(\mathrm{SU}_{3}\right) \times \mathrm{E}_{6} \supset\left(\mathrm{SU}_{3}\right) \times \mathrm{SU}_{2} \times \mathrm{SU}_{6} \\
& \supset\left(\mathrm{SU}_{3}\right) \times \mathrm{SU}_{2} \times \mathrm{U}_{1}^{Y^{\prime}} \times \mathrm{SU}_{5}, \\
\mathrm{E}_{8} & \supset \mathrm{SO}_{6} \times \mathrm{SO}_{10} \supset\left(\mathrm{SU}_{3}\right) \times \mathrm{U}_{1} \times \mathrm{SO}_{10} \\
& \supset\left(\mathrm{SU}_{3}\right) \times \mathrm{U}_{1} \times \mathrm{U}_{1}^{B-L} \times \mathrm{SU}_{5},
\end{aligned}
$$

where $\mathrm{U}_{1} \times \mathrm{SO}_{10} \subset \mathrm{E}_{6}$ and $\mathrm{SO}_{6} \sim \mathrm{SU}_{4}$. Thus, concerning the phenomenology of the observable gauge sector, it is enough, as it is usualy done, to concentrate on $\mathrm{E}_{6}$ compactifications and the relax some $\mathrm{E}_{6}$ constraints [6]. On different grounds, a similar result was argued in ref. [7].

It is noticeable that if only one new gauge boson were found, and it could not be associated with one of these $U_{1}$, superstring unification should be abandoned. On the contrary, if such a $U_{1}$ were established, the superstring scenario would be the likely origin. The former analysis is general, but there is a subtlety in the case that at the last step of symmetry breaking down to the standard model, there is more than one $U_{1}$. In this case, the lightest gauge boson can couple to any current which is a linear combination of the extra $U_{1}$ 's, but then at least a second light (gauge) boson should be found. Still one of the extra $U_{1}$ 's has to be one of the former two. Moreover, it can be proven on similar grounds that if there are more than one extra $U_{1}$ 's, two of them must be $Y^{\prime}$ and $B-L$, or only one of them but then a second $\mathrm{U}_{1}$ must live in $\mathrm{SO}_{12}, \mathrm{E}_{8} \supset \mathrm{SO}_{4} \times \mathrm{SO}_{12}$. (However, $\mathrm{SO}_{12}$ has only selfconjugate representations and does not allow for chiral families.) In that case the lightest extra neutral gauge boson will not coincide in general with the extra gauge boson predicted by theories with only one extra $U_{1}$ at low energies though in some particular cases it might do. To ilustrate this statement, let us assume that the two extra $U_{1}$ in $E_{6}$ are unbroken, and that the multiplicatively renormalized gauge bosons correspond to the extra hypercharge $Y^{\prime}$ in the minimal model and the remaining

\footnotetext{
${ }^{\# 1}$ For a detailed discussion of $E_{6}$ and $E_{8}$ see ref. [5].
}

orthogonal hypercharge $Y^{\prime \prime}$ in $\mathrm{E}_{6}$. (We follow the notation nd conventions of ref. [8].) If they are broken at some scale along the fundamental $E_{6}$ representation, 27, the massive boson mass matrix is proportional to

$$
\begin{array}{cc}
Y^{\prime} & Y^{\prime \prime} \\
Y^{\prime} & \left.\begin{array}{cc}
\frac{5}{3} \tilde{g}^{\prime 2}(a+b) & \sqrt{\frac{5}{3}} \tilde{g}^{\prime} \tilde{g}^{\prime \prime}(a-b) \\
\sqrt{\frac{5}{3}} \tilde{g}^{\prime} \tilde{g}^{\prime \prime}(a-b) & \tilde{g}^{\prime \prime 2}(a+b)
\end{array}\right),
\end{array}
$$

where $a(b)=\left|\left\langle\nu_{5(4)}\right\rangle\right|^{2}$ are the vacuum expectation values along the neutral directions, $\left(Y_{\nu_{5.4}}^{\prime}, Y_{\nu_{S, 4}}^{\prime \prime}\right)=\left(\frac{1}{2} \sqrt{\frac{5}{3}}, \pm \frac{1}{2}\right) . \quad$ In the case $a=b$, $5 \tilde{g}^{\prime 2}<3 \tilde{g}^{\prime \prime 2}$, the lightest gauge boson is that of the minimal model, which is usually associated to flux breaking $[9,10]$. For an arbitrary set of values, $a, b$, $\tilde{g}^{\prime} / \tilde{g}^{\prime \prime}$, the lightest gauge boson can couple to an arbitrary combination of $Y^{\prime}, Y^{\prime \prime}$, but then another light gauge boson must also exist and be found. If $a \gg b$ we recover $B-L$, and the applicability of our analysis ( $b \gg a$ corresponds also to $B-L$, after a proper redefinition of matter fields [8]). In short, if an extra gauge boson fitting one of the models (below) with an extra $U_{1}$ were found at accelerator energies then superstring theories would be strengthened, although we could not exclude in general the existence of a second extra gauge boson. If an extra gauge boson fitting no model with an extra $U_{1}$ were found, then either an additional (heavier) gauge boson exists in the $\mathrm{TeV}$ range or superstring theories are ruled out. Needless to say that if no extra gauge boson is found, superstring theories are neither confirmed nor ruled out.

With our present understanding of string theories, no preferred extension of the SM can be singled out. In general, all of them predict new matter fields at low energy and, due to supersymmetry, a plethora of new particles should be discovered in future experiments. Proton decay and neutrino masses are constant problems. However, string theories allow for (discrete) symmetries, similar to those required for light higgses, to control these diseases [6]. Extended electroweak models are constrained by experiment, but they are not excluded $[8,10]$. Nevertheless, in order to account for the correct (vanishing) Yukawa couplings and non-renormalizable terms in the effective low-energy lagrangian, superstring theories must be very powerful and master our taste about natu- 
ralness. In this paper we assume the existence of extra weak interactions from the heterotic string. We then calculate the renormalization group predictions for the mixing angles defining the new gauge currents, and we observe that their measurement can determine at large extent the superstring model at the compactification scale. We present full details and a complete analysis elsewhere [11].

As we argued before, we can restrict ourselves to $\mathrm{E}_{6}$ but relaxing some constraints. The fermionic neutral lagrangian is enough to fix the gauge interactions $[8,12,13]$,

$$
\begin{aligned}
& \mathscr{L}_{\mathrm{NC}}=e J_{\mathrm{EM}}^{\mu} A_{\mu}+\frac{e}{s_{\mathrm{w}} c_{\mathrm{w}}} J_{\mathrm{Z}}^{\mu} / Z_{\mu}+\frac{e}{c_{\mathrm{w}}} J_{\mathrm{Z}^{\prime}}^{\mu} \cdot Z_{\mu}^{\prime}, \\
& J_{\mathrm{EM}}=J_{1}+\sqrt{\frac{5}{3}} J_{2}, \\
& J_{\mathrm{Z}}=c_{3} J_{\mathrm{Z}^{0}}-s_{3} J_{\mathrm{Z}^{0}}, \quad J_{\mathrm{Z}^{\prime}}=s_{3} J_{\mathrm{Z}^{0}}+c_{3} J_{\mathrm{Z}^{0}}, \\
& J_{\mathrm{Z}^{0}}=J_{1}-s_{\mathrm{W}}^{2} J_{\mathrm{EM}}, \\
& J_{\mathrm{Z}^{0}}=\sqrt{\frac{5}{3}}\left[s_{1} c_{1}(\lambda-1 / \lambda) J_{2}\right. \\
& \left.\quad+\left(c_{1}^{2} / \lambda+\lambda s_{1}^{2}\right)\left(-c_{2} J_{3}+s_{2} J_{4}\right)\right]
\end{aligned}
$$

where $A, Z, Z^{\prime}$ correspond to the photon, the observed $\mathrm{Z}$ and the new gauge boson, respectively. $e$ is the electromagnetic constant, $s_{\mathrm{w}}\left(c_{\mathrm{w}}\right)$ the sinus cosinus) of the Weinberg angle and $J_{i}^{\mu}$ the $\mathrm{E}_{6}$ currents. $\left(J_{i}^{\mu}=\bar{\psi} T_{i} \gamma^{\mu} \psi\right.$ are the fermionic currents, with $\left(T_{i}\right)=\left(T_{3 \mathrm{~L}}, Y, Y^{\prime} Y^{\prime \prime}\right)$ an orthogonal basis of generators, explicitly given in ref. [8]). $c_{i}\left(s_{i}\right)=\cos \theta_{i}$ $\left(\sin \theta_{i}\right)$, where $\theta_{3}$ gives the $\mathrm{Z}^{0} \mathrm{Z}^{\prime}$ mixing. This is known to be small [12]. It mainly depends on the ratios of the different vacuum expectation values, and will be strongly constrained in forthcoming experiments. $\theta_{2}$ can take, up to a degeneracy, only two values [8], corresponding to the two different extra $U_{1}$ at low energy. Finally $\theta_{1} \in\left[-\frac{1}{2} \pi, \frac{1}{2} \pi\right)$ and $\lambda=\tilde{g}_{2} / \tilde{g}_{3} \geqslant 1$ (a ratio of coupling constants) are the two parameters probing the high-energy region through renormalization effects, and the ones we want to calculate. They could be measured in production experiments $[8,12,13]$. If so, they would at large extent fix the string model at the compactification scale.

We work at one loop. Two-loop contributions should be small, and due to the large uncertainties, it appears unnecessary to consider them in general. If they were large, perturbation theory would loose
Table 1

$\begin{array}{lll}\text { (i) } \mathrm{G} \rightarrow \mathrm{SU}_{3}^{\mathrm{C}} \times \mathrm{SU}_{2}^{\mathrm{L}} \times \mathrm{U}_{1}^{\gamma} \times \mathrm{U}_{1} & \\ \text { B3: } & \mathrm{SU}_{4}^{\mathrm{C}} \times \mathrm{SU}_{2}^{\mathrm{L}} \times \mathrm{SU}_{2}^{Y^{\prime \prime}} \times \mathrm{U}_{1} & \left\langle\nu_{4,5}\right\rangle \\ \mathrm{B} 4: & \mathrm{SU}_{3}^{\mathrm{C}} \times \mathrm{SU}_{3}^{\mathrm{L}} \times \mathrm{SU}_{2}^{Y \prime \prime} \times \mathrm{U}_{1} & \left\langle\nu_{4,5}\right\rangle \\ \mathrm{B} 7: & \mathrm{SU}_{4}^{\mathrm{C}} \times \mathrm{SU}_{2}^{\mathrm{L}} \times \mathrm{SU}_{2}^{\mathrm{R}} \times \mathrm{U}_{1} & \left\langle\nu_{4}\right\rangle \\ \text { B8: } & \mathrm{SU}_{3}^{\mathrm{C}} \times \mathrm{SU}_{3}^{\mathrm{L}} \times \mathrm{SU}_{2}^{\mathrm{R}} \times \mathrm{U}_{1} & \left\langle\nu_{4}\right\rangle \\ \mathrm{B} 13: & \mathrm{SU}_{5}^{\mathrm{CL}} \times \mathrm{U}_{1}^{\mathrm{R}} \times \mathrm{U}_{1} & \left\langle\nu_{5}\right\rangle \\ \mathrm{C} 1: & \mathrm{SU}_{4}^{\mathrm{C}} \times \mathrm{SU}_{2}^{\mathrm{L}} \times \mathrm{U}_{1}^{Y^{\prime \prime}} \times \mathrm{U}_{1} & \left\langle\nu_{4,5}\right\rangle \\ \mathrm{C} 2: & \mathrm{SU}_{3}^{\mathrm{C}} \times \mathrm{SU}_{3}^{\mathrm{L}} \times \mathrm{U}_{1}^{Y^{\prime \prime}} \times \mathrm{U}_{1} & \left\langle\nu_{4,5}\right\rangle \\ \mathrm{C} 3: & \mathrm{SU}_{3}^{\mathrm{C}} \times \mathrm{SU}_{2}^{\mathrm{L}} \times \mathrm{SU}_{2}^{Y^{\prime \prime}} \times \mathrm{U}_{1}^{Y} \times \mathrm{U}_{1}^{Y} & \left\langle\nu_{4}\right\rangle \\ \mathrm{C} 4: & \mathrm{SU}_{4}^{\mathrm{C}} \times \mathrm{SU}_{2}^{\mathrm{L}} \times \mathrm{U}_{1}^{\mathrm{R}} \times \mathrm{U}_{1} & \left\langle\nu_{4}\right\rangle \\ \mathrm{C} 5: & \mathrm{SU}_{3}^{\mathrm{C}} \times \mathrm{SU}_{2}^{\mathrm{L}} \times \mathrm{SU}_{2}^{\mathrm{R}} \times \mathrm{U}_{1} \times \mathrm{U}_{1} & \left\langle\nu_{4}\right\rangle \\ \mathrm{D}: & \mathrm{SU}_{3}^{\mathrm{C}} \times \mathrm{SU}_{2}^{\mathrm{L}} \times \mathrm{U}_{1}^{Y} \times \mathrm{U}_{1}^{Y^{\prime}} \times \mathrm{U}_{1}^{Y^{\prime \prime}} & \left\langle\nu_{4,5}\right\rangle\end{array}$

(ii) $\mathrm{G} \rightarrow \mathrm{SU}_{3}^{\mathrm{C}} \times \mathrm{SU}_{2}^{\mathrm{L}} \times \mathrm{SU}_{2}^{\mathrm{R}} \times \mathrm{U}_{1}$

$\begin{array}{lll}\mathrm{A} 4: & \mathrm{SU}_{3}^{\mathrm{C}} \times \mathrm{SU}_{3}^{\mathrm{L}} \times \mathrm{SU}_{3}^{\mathrm{R} Y^{n}} & \left\langle\nu_{4,5}\right\rangle \\ \mathrm{B} 6: & \mathrm{SU}_{5}^{\mathrm{CL}} \times \mathrm{SU}_{2}^{\mathrm{R}} \times \mathrm{U}_{1} & \left\langle\nu_{4}\right\rangle \\ \mathrm{B} 8: & \mathrm{SU}_{3}^{\mathrm{C}} \times \mathrm{SU}_{2}^{\mathrm{L}} \times \mathrm{SU}_{2}^{\mathrm{R}} \times \mathrm{U}_{1} & \left\langle\nu_{5}\right\rangle \\ \mathrm{B} 9: & \mathrm{SU}_{5}^{\mathrm{CR}} \times \mathrm{SU}_{2}^{\mathrm{L}} \times \mathrm{U}_{1} & \left\langle\nu_{5}\right\rangle \\ \text { B11: } & \mathrm{SU}_{3}^{\mathrm{C}} \times \mathrm{SU}_{3}^{\mathrm{R}} \times \mathrm{SU}_{2}^{\mathrm{L}} \times \mathrm{U}_{1} & \left\langle\nu_{5}\right\rangle \\ \mathrm{C} 5: & \mathrm{SU}_{3}^{\mathrm{C}} \times \mathrm{SU}_{2}^{\mathrm{L}} \times \mathrm{SU}_{2}^{\mathrm{R}} \times \mathrm{U}_{1} \times \mathrm{U}_{1} & \left\langle\nu_{5}\right\rangle\end{array}$

(iii) $\mathrm{SU}_{3}^{\mathrm{C}} \times \mathrm{SU}_{2}^{\mathrm{L}} \times \mathrm{U}_{1}^{Y} \times \mathrm{U}_{1}^{Y^{\prime}}$

(iv) $\mathrm{SU}_{3}^{\mathrm{C}} \times \mathrm{SU}_{3}^{\mathrm{L}} \times \mathrm{U}_{1}$

its applicability, and then even one-loop corrections could not be trusted. To classify the different possibilities we follow closely the analysis of ref. [14]. More details are given elsewhere [11]. $\mathrm{E}_{6}$ extended electroweak models with one extra neutral gauge boson can be of rank 5 or 6 at the compactification scale. If they are of rank 6 , they must allow for a large intermediate scale. In this case the low-energy group ( $\sim 1 \mathrm{TeV}$ ) can be (assuming that the vacuum mainly aligns along fundamental $E_{6}$ representations) $\mathrm{SU}_{3}^{\mathrm{C}} \times \mathrm{SU}_{2}^{\mathrm{L}} \times \mathrm{U}_{1}^{Y} \times \mathrm{U}_{1}$ or $\mathrm{SU}_{3}^{\mathrm{C}} \times \mathrm{SU}_{2}^{\mathrm{L}} \times \mathrm{SU}_{2}^{\mathrm{R}} \times \mathrm{U}_{1} \# 2$. Limits on proton decay, neutrino masses and rare processes, in particular $\mathrm{K} \rightarrow \mu \mathrm{e}$, exclude other possibilities $[11,14]$. The $E_{6}$ subgroups of rank 6 which reduce to them are collected in table 1 (i), (ii). The allowed (large) vacuum directions correspond to $\nu_{4,5}$. The broken direction is given in brackets. Left-right (LR) models require $\nu_{5}$ to be the non-zero vacuum direction. When $\nu_{4,5}$ give equivalent results, both are in brackets. For easier contact with ref. [11], we

\#2 C stands for color, $\mathrm{SU}_{2}^{\mathrm{L}} \times \mathrm{U}_{1}^{Y}$ is the standard model and $\mathrm{R}$ corresponds to the usual right-handed current [15]. 
enumerate the different cases in the same way as we do there $(B 3, B 4, \ldots)$, although here it looks weird, for only some groups are of interest. On similar grounds, the groups of rank 5 at the compactification scale which can survive down to low energies are, $\mathrm{SU}_{3}^{\mathrm{C}} \times \mathrm{SU}_{2}^{\mathrm{L}} \times \mathrm{U}_{1}^{Y} \times \mathrm{U}_{1}^{Y^{\prime}} \quad$ (iii) and $\mathrm{SU}_{3}^{\mathrm{C}} \times \mathrm{SU}_{3}^{\mathrm{L}} \times \mathrm{U}_{1}$ (iv). Given the gauge group, the model is specified once the matter content is fixed. We assume throughout the paper a supersymmetric spectrum down to $1 \mathrm{TeV}$.

Let us first discuss the predictions for the rank 6 groups with a large intermediate scale. Usually the strong and the electromagnetic coupling constants $\alpha_{3}\left(M_{Z}\right)=0.12$ and $\alpha_{\mathrm{E}}\left(M_{\mathrm{Z}}\right)=(128)^{-1},\left(\alpha=g^{2} / 4 \pi\right.$ and $\left.M_{\mathrm{Z}}=92 \mathrm{GeV}\right)$ are input parameters. Then unification fixes the Weinberg angle [16]. We use, however, this as an input parameter, $\sin ^{2} \theta_{\mathrm{w}}=0.225 \pm 0.015$, and then unification fixes the intermediate scale. Which in turn fixes the renormalization of $\theta_{1}$ and $\lambda$. The results are given in fig. 1 for the minimal electroweak extension $S_{3}^{\complement} \times$ $\mathrm{SU}_{2}^{\mathrm{L}} \times \mathrm{U}_{1}^{Y} \times \mathrm{U}_{1}$ in table 1 (i). Here, we assume the minimal matter content at the compactification scale: 3 complete 27 plus the components of $27+\overline{27}$ where the field directions acquiring a large VEV lie. We then assume that $(\overline{27})^{3}$ couplings give masses to all possible fields except for the standard Higgses at the intermediate scale $[10,14,17,18]$

$M_{1} \sim\left(M_{\mathrm{s}} / \lambda_{n}\right)^{1 /(2 n-2)} M_{\mathrm{P} 1}^{(2 n-3) /(2 n-2)}$,

where $M_{\mathrm{s}}$ is the effective scale of supersymmetry breaking along the flat direction, $\lambda_{\mathrm{n}}$ the coupling of the non-renormalizable term $(27 \overline{27})^{n}$ lifting the flatness and $M_{\mathrm{P} 1}$ the Planck mass. These couplings will provide a common mass $M_{\mathrm{s}}$ to all survivors of $27+\overline{27}$. The new gauge boson and the supersymmetric particles (except the standard higgsinos) get masses at $1 \mathrm{TeV}$. Below $1 \mathrm{TeV}$ we only consider the three chiral families of standard fermions and the standard higgses and higginos. (Light SM neutrals do not contribute at this state.) Multiplet assignments and $\beta$ functions are given in ref. [11], as well as an exhaustive numerical analysis of different matter contents. Lines in fig. 1 can stop in between $s_{\mathrm{w}}^{2}=0.21$ and $s_{\mathrm{w}}^{2}=0.24$ because we do not find perturbative unification between $10^{15-20} \mathrm{GeV}$, because a Landau pole is reached before unification or

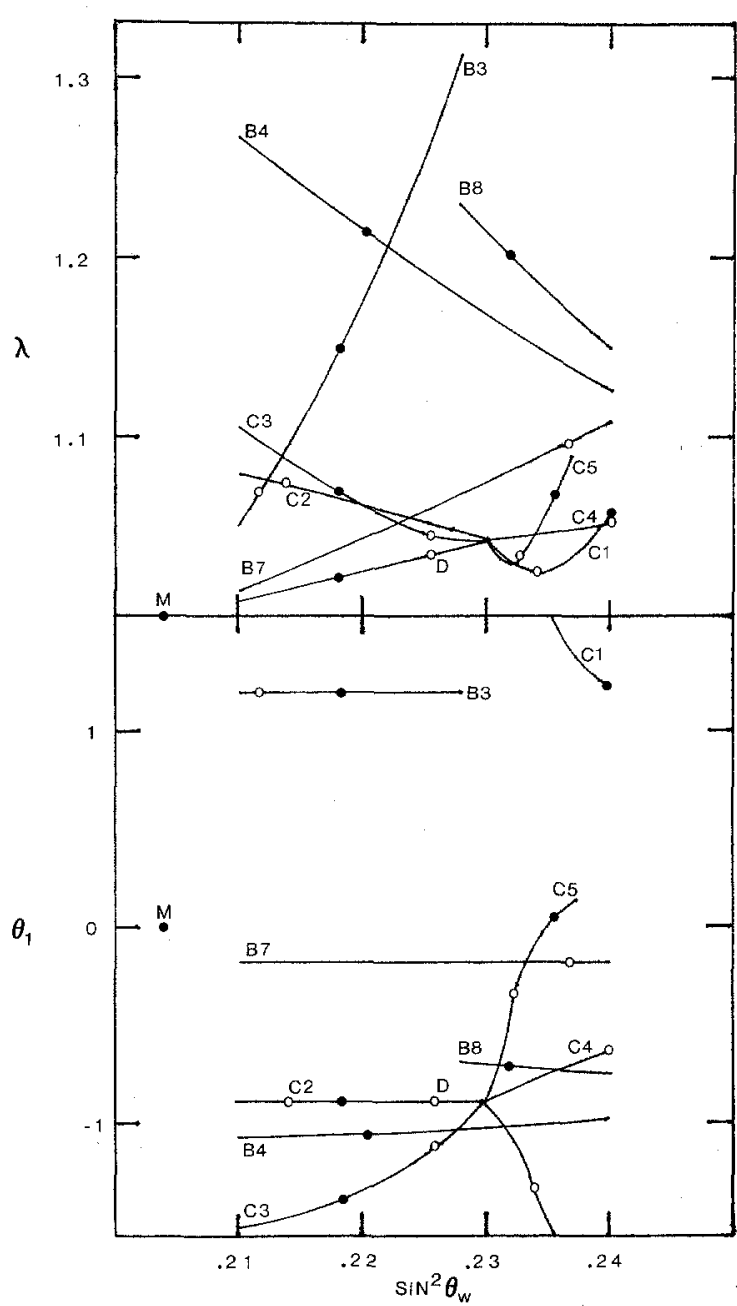

Fig. 1.

because $M_{\mathrm{I}}\left(\leqslant M_{\mathrm{X}}\right)$ is below $10^{4} \mathrm{GeV}$. In table 2 we give for the different cases, and as a function of $s_{\mathrm{w}}^{2}$, the initial and final values of $M_{\mathrm{I}}, M_{\mathrm{X}}$ and $\alpha_{\mathrm{X}}$, where $\mathrm{X}$ (I) stands for the compactification (intermediate) scale. Their dependence on $s_{w}^{2}$ interpolates almost logarithmically between their extreme values. Parameters satisfying the limiting conditions are outlined. Although we allow for any intermediate scale between $10^{4} \mathrm{GeV}$ and $M_{\mathrm{X}}$, it is expected to be typically $\sim 10^{10} \mathrm{GeV}$ (a value which we mark with dots in fig. 1), see eq. (6), or $10^{14} \mathrm{GeV}$ if dangerous proton decay operators of dimension five are present $[11,14]$. Similarly $M_{\mathrm{X}} \sim 10^{17-19} \mathrm{GeV}$. As we ignore renormalization effects due to the spreading of mat- 


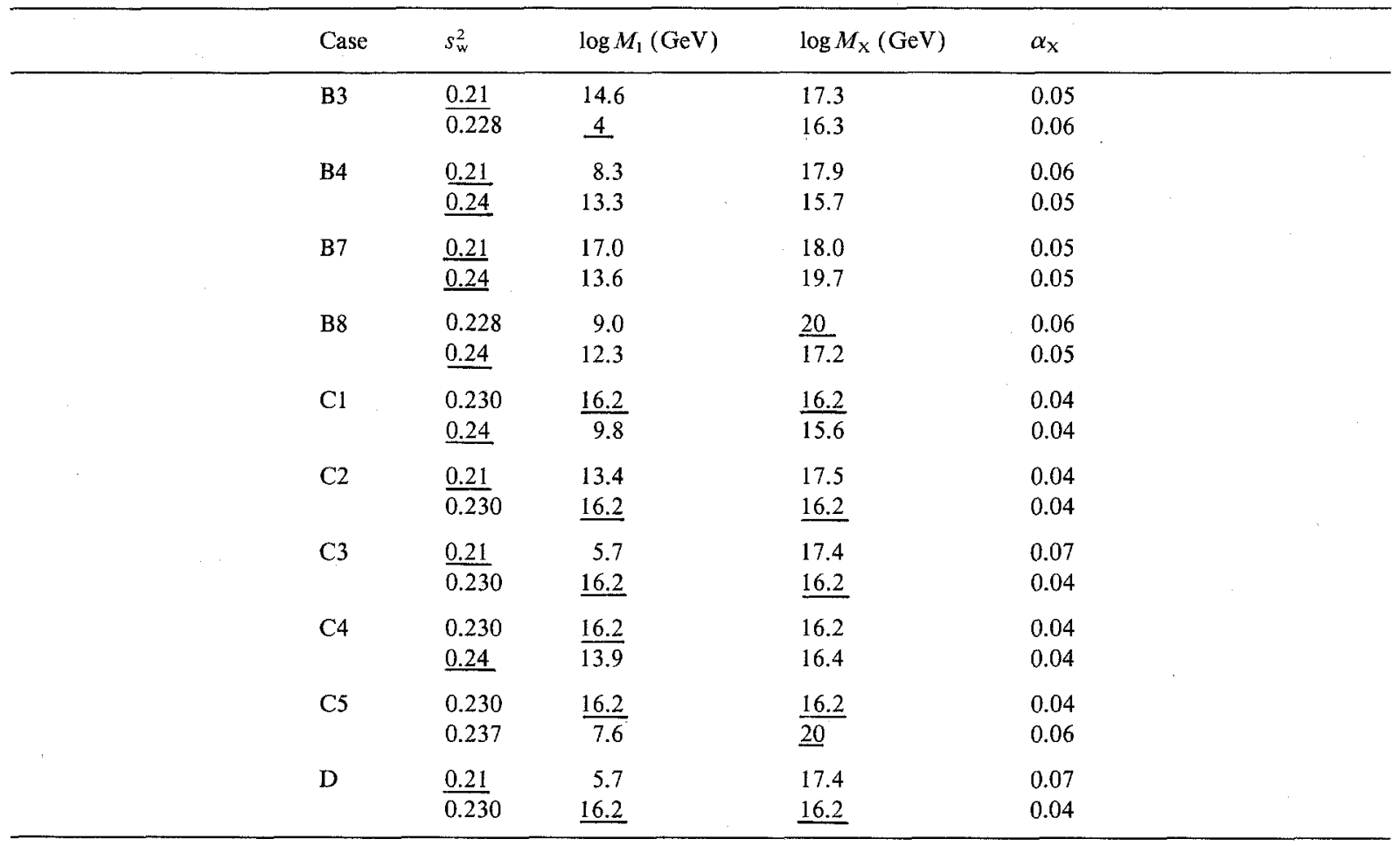

ter masses around each scale (to make the problem tractable), we effectively use $M_{\mathrm{I}}$ as a free parameter. For $M_{\mathrm{I}}$ very large the compactification might be disturbed, but we are only concerned with possible renormalization effects. For non-standard $\mathrm{SU}_{5}$ unification, B13 in table 1(i), $M_{\mathbf{I}}$ must be very large to avoid too large a rate for proton decay. Anyway, we do not find any $M_{1}$ fulfilling all requirements in this case. For the other models we always find solutions, but in cases B3, B7, C4, only those with $M_{\mathrm{I}}>10^{14}$ $\mathrm{GeV}$ are allowed in order to banish proton decay within experimental bounds. The circles in fig. 1 correspond to $M_{\mathrm{I}}=10^{14} \mathrm{GeV}$, and they separate the excluded region from the allowed one in those cases. One may wonder about the meaning of the common point, $\sin ^{2} \theta_{\mathrm{w}}=0.23, \theta_{1}=-0.9, \lambda=1.04$, for cases $\mathrm{C} 1$, $\mathrm{C} 2, \ldots, \mathrm{C} 5, \mathrm{D}$. It corresponds to the case in which the intermediate and the unification scales coincide $\left(\sim 10^{16.2} \mathrm{GeV}\right)$. Then the renormalization results from evolving the $\mathrm{SU}_{3}^{\mathrm{C}} \times \mathrm{SU}_{2}^{\mathrm{L}} \times \mathrm{U}_{1}^{Y} \times \mathrm{U}_{1}$ coupling constants in the presence of three families of $16+1$ elements.
Since the $E_{6}$ representations are not complete at each energy scale, the multiplicatively renormalized gauge bosons do not coincide in general with those chosen conventionally. Then, rotations must be done at each stage to diagonalize the matrix of $U_{1} \beta$-functions as well as to take into account the symmetry breaking. There is no simple general expression for them, and we must then perform a numerical analysis [11].

For the LR models at low energy, (ii) in table 1, we have performed a similar analysis. We find, as is well known $[1,14]$ that no model gives the correct value for the Weinberg angle (assuming unification near the Planck scale), but rather values that are too large. Spreading the masses around the different mass scales could improve the predictions, in particular the supersymmetric spreading around $1 \mathrm{TeV}$ could have an effect. Numerically the only general possibility to recover phenomenologically these models seems to rely on the relaxation of the unification hypothesis at the compactification scale. (Which is our working ssumption and which allows for pre- 
dictions.) This has been proposed in standard grand unified theories [19], where it was argued that gravitational effects could change standard predictions. In string theories the unification of coupling constants occurs at tree level [6]. It is reasonable, however, that if there is not a real gauge unification at the compactification scale, the same mechanism spoiling the gauge unification may renormalize the coupling constants differently. There is a claim that such effects must exist at higher order in the loop expansion of the string theory [20] and in particular, that string loop corrections can be large [21]. We studied numerically the necessary spreading of coupling constants at the compactification scale for $L R$ models at low energy elsewhere [11]. (Contributions from extra matter were also considered.)

All models in table 1 (i), (ii) contain after symmetry breaking $T_{3}^{\mathrm{L}}, T_{3}^{\mathrm{R}}$ and $B-L$, the diagonal generators of $\mathrm{SU}_{2}^{\mathrm{L}} \times \mathrm{SU}_{2}^{\mathrm{R}} \times \mathrm{U}_{1}^{B-L} \quad[11,15]$. Strictly speaking, with the conventional assignments of matter fields this is so only when the vacuum aligns along $\left\langle\nu_{5}\right\rangle$. The case $\left\langle\nu_{4}\right\rangle \neq 0$ differs by a rotation which can be absorbed in a redefinition of the matter fields [8]. $\left\langle\nu_{5,4}\right\rangle$ always leave an $\mathrm{SO}_{10}$ unbroken and the resulting group is the intersection of this $\mathrm{SO}_{10}$ and the corresponding rank 6 subgroup of $E_{6}$. If this group distinguishes between both directions then both cases have to be analysed separately, in particular the renormalization effects we consider are different. In all models $\left(c_{2}, s_{2}\right)=(\sqrt{3 / 8}, \sqrt{5 / 8})$ in eq. (5) (but for $\left\langle\nu_{4}\right\rangle$ breaking, however, a rotation, not relevant here, must accompany the matter fields if the standard assignments are going to be maintained).

Finally we calculate the mixing angle(s) characterizing the models of rank 5 at the compactification scale, $\mathrm{SU}_{3}^{\mathrm{C}} \times \mathrm{SU}_{3}^{\mathrm{L}} \times \mathrm{U}_{1}$ and $\mathrm{SU}_{3}^{\mathrm{C}} \times \mathrm{SU}_{2}^{\mathrm{L}} \times \mathrm{U}_{1}^{Y} \times \mathrm{U}_{1}^{Y^{\prime}}$. The assumptions are the same as before but now there is no large intermediate scale. $\left(c_{2}, s_{2}\right)=(0,1)$ in eq. (5) for both cases. For the minimal model $\mathrm{SU}_{3}^{C} \times$ $\mathrm{SU}_{2}^{\mathrm{L}} \times \mathrm{U}_{1}^{Y} \times \mathrm{U}_{1}^{Y^{\prime}}$ [10] in table 1 (iii) we find $s_{\mathrm{w}}^{2}=0.204, M_{\mathrm{X}}=10^{17.7}, \alpha_{\mathrm{X}}=0.09, \theta_{1}=0, \lambda=1(\mathrm{M}$ in fig. 1). That is too low a Weinberg angle for present experimental limits $[8,22]$. The addition of extra matter can increase the value of the Weinberg angle but lowers the value of the unification scale [11]. Model (iv) in table $1, \mathrm{SU}_{3}^{\mathrm{C}} \times \mathrm{SU}_{3}^{\mathrm{L}} \times \mathrm{U}_{1}$, does not unify as $\mathrm{SU}_{3}^{\mathrm{C}, \mathrm{L}}$ couplings run parallel. But unification can be reached adding extra matter, although at the cost of too low a mass scale and too low a Weinberg angle. Relaxing the unification hypothesis, many case are recovered.

In conclusion, we have shown that if a new gauge boson exist around $1 \mathrm{TeV}$, its interactions, if coming from superstrings, are essentially fixed by two parameters $\theta_{1}, \lambda$. It is worth emphasizing that the angle $\theta_{1}$, which comes essentially from renormalization effects, should not be mistaken for $\theta_{2}$ in eq. (5), which is group theoretically fixed by the vacuum alignment. Actually, $\theta_{1}$ can take any value, between $-\frac{1}{2} \pi$ and $\frac{1}{2} \pi$, as can be seen in fig. 1 , while $\theta_{2}$ can only take two values in (heterotic) superstring models if only one extra gauge boson is present at low energy. We present the renormalization group predictions for $\theta_{1}, \lambda$, as well as for $\sin ^{2} \theta_{\mathrm{w}}, \ldots$, (assuming the minimal matter content) and show that their measurement can provide crucial information on the model at the compactification scale. The results are collected in fig. 1 and table 2 for models with a large intermediate scale and one extra $\mathrm{U}_{1}$ at $1 \mathrm{TeV}$. LR models use to give too large a Weinberg angle, whereas models of rank 5 at the unification scale give a low Weinberg angle ( $M$ in fig 1$)$ or do not unify. Extra matter (in incomplete $27+\overline{27})$ spreads the range of variation of the different parameters. The possible additions are described elsewhere [11]. If no unification is assumed, any set of parameters can be in general obtained at low energy by properly choosing the initial conditions at the compactification scale.

We thank G. Kane for a careful reading of the manuscript and P. Ramond, G.G. Ross and F. Zwirner for valuable discussions. One of us (F.A.) acknowledges the stimulating atmosphere and kind hospitality extended to him when visiting the Physics Department at the University of Florida, where this work was partially done. We borrowed the inspiration for the title of this paper from ref. [23]. We hope new neutral currents to be less elusive than has been proton decay so far.

\section{References}

[1] M. Green, J. Schwarz and E. Witten, Superstring theory (Cambridge U.P., Cambridge, 1987), and references therein. 
[2] D. Gross, J. Harvey, E. Martinec and R. Rohm, Phys. Rev. Lett. 54 (1985) 502; Nucl. Phys. B 256 (1985) 253; B 267 (1986) 75 .

[3] K.S. Narain, Phys. Lett. B 169 (1986) 41;

K.S. Narain, M.H. Sarmadi and E. Witten, Nucl. Phys. B 279 (1987) 369;

I. Antoniadis, C. Bachas and C. Kounnas, LBL preprint LBL 22709 (1986);

L. Ibáñez, P. Nilles and F. Quevedo, Phys. Lett. B 187 (1987) 25 ;

H. Kawai, D.C. Lewellen and S.H.H. Tye, Cornell preprint CLNS 86/751.

[4] F. Gursey, P. Ramond and P. Sikivie, Phys. Lett. B 60 (1976) 177 ;

Y. Achiman and B. Stech, Phys. Lett. B 77 (1978) 389;

Q. Shafi, Phys. Lett. B 79 (1978) 301;

H. Ruegg and T. Schucker, Nucl. Phys. B 161 (1979) 388;

R. Barbieri and D.V. Nanopoulos, Phys. Lett. B 91 (1980) 369.

[5] R. Slansky, Phys. Rep. 79 (1981) 1.

[6] E. Witten, Nucl. Phys. B 258 (1985) 75.

[7] B. Campbell, J. Ellis and D.V. Nanopoulos, Phys. Lett. B $181(1986) 283$.

[8] F. del Aguila, M. Quiros and F. Zwirner, Nucl. Phys. B 287 (1987) 419; see also F. del Aguila, G. Blair, M. Daniel and G.G. Ross, Nucl. Phys. B 283 (1987) 50.

[9] Y. Hosotani, Phys. Lett. B 126 (1983) 309; B 129 (1983) 193 ;

E. Witten, Phys. Lett. B 149 (1984) 351.
[10] E. Cohen, J. Ellis, K. Enqvist and D.V. Nanopoulos, Phys. Lett. B 165 (1985) 76;

J. Ellis, K. Enqvist, D.V. Nanopoulos and F. Zwirner, Nucl. Phys. B 276 (1986) 14; Mod. Phys. Lett. A 1 (1986) 57.

[11] F. del Aguila, J Gonzalez and M. Quiros, UAB preprint, UAB-FT-167 (1987).

[12] F. del Aguila, M. Quiros and F. Zwirner, Nucl. Phys. B 284 (1987) 530.

[13] F. del Aguila, E. Laermann and P. Zerwas, UF preprint UFTP-87-11 (1987).

[14] M. Dine, V. Kaplunovsky, M. Mangano, C. Nappi and N. Seiberg, Nucl. Phys. B 259 (1985) 549.

[15] A. De Rujula, H. Georgi and S.L. Glashow, Ann. Phys. (NY) 109 (1977) 242, 258;

R.N. Mohapatra and D.P. Sidler, Phys. Rev. Lett. 38 (1977) 665.

[16] H. Georgi, H.R. Quinn and S. Weinberg, Phys. Rev. Lett. 33 (1974) 451.

[17] F. del Aguila, G. Blair, M. Daniel and G.G. Ross, Nucl. Phys. B 272 (1986) 413.

[18] G. Costa, F. Feruglio, F. Gabbiani and F. Zwirner, Nucl. Phys. B 286 (1987) 325.

[19] C.T. Hill, Phys. Lett. B 135 (1984) 47.

[20] K. Choi and J.E. Kim, Phys. Lett. B 165 (1985) 71; B 176 (1986) 103.

[21] K. Choi, HUTP preprint HUTP-87/A004 (1987).

[22] U. Amaldi et al., UP preprint UPR-0331T (1987);

G. Costa, J. Ellis, G.L. Fogli, D.V. Nanopoulos and F. Zwirner, CERN preprint TH-4675/87 (1987).

[23] A. De Rujula, H. Georgi and S.L. Glashow, Phys. Rev. Lett. $45(1980) 413$. 